\title{
Utility of immunohistochemical expression of E-cadherin in colorectal carcinoma
}

\author{
Sharmilla Devi Rajkumar ${ }^{1}$ Barathi Gunabooshanam², Leena Dennis Joseph², Lawrence D’Cruze ${ }^{2}$ \\ ${ }^{1}$ SRM Institute of Medical Sciences, Chennai, India \\ 2Department of Pathology, Sri Ramachandra Medical College, Sri Ramachandra Institute of Higher Education and Research, \\ Chennai, India
}

Gastroenterology Rev 2022; 17 (1): 59-66

DOI: https://doi.org/10.5114/pg.2021.107801

Key words: colorectal cancer, E-cadherin, immunohistochemistry, prognosis.

Address for correspondence: Barathi Gunabooshanam MD, DNB, DCP, Assistant Professor, Department of Pathology, Sri Ramachandra Medical College, Sri Ramachandra Institute of Higher Education and Research, Chennai, India, e-mail: gbarathi@sriramachandra.edu.in

\begin{abstract}
Introduction: Reduced expression of E-cadherin, an intercellular junction protein, is associated with differentiation and metastasis of multiple cancers, including colorectal cancer.

Aim: To investigate the utility of the immunohistochemistry of E-cadherin as a prognostic marker for colorectal cancer (CRC).

Material and methods: Immunohistochemical analysis for E-cadherin was performed on 100 paraffin blocks retrieved from resected specimens of CRC patients. The collected data were statistically analysed.

Results: Among the 100 patients, men comprised $58 \%$ and the majority had tumour size of $5-10 \mathrm{~cm}(55 \%)$. Grade II CRC was more common (74\%) than grade I and III (13\% each). The correlation of E-cadherin expression with lymph node involvement was statistically significant, as revealed by $p$-value $<0.01$, with about $27 \%$ in N1 and $13 \%$ in N2 stage. E-cadherin expression was significantly correlated with tumour differentiation pattern $(p<0.01)$, wherein out of 13 poorly differentiated carcinomas, $38.5 \%$ and $30.5 \%$ of samples showed negative and weak E-cadherin staining, respectively.

Conclusions: Furthermore, a shift from membranous E-cadherin staining in normal cells to cytoplasmic and mixed staining was observed in cancer cells. The study indicates that immunohistochemical E-cadherin expression has prognostic value, as revealed by its loss of expression in poorly differentiated cells and lymph node metastasis.
\end{abstract}

\section{Introduction}

E-cadherin is a type I classical cadherin, which mediates calcium-dependent cell-cell adhesion and regulates multiple cellular functions like proliferation, differentiation, migration, and polarity [1]. In colonic mucosa $\mathrm{E}$-cadherin is expressed on the basolateral aspects of the cell membrane, in all crypt and surface epithelial cells, establishing in this way mucosal integrity. The abnormal expression and function of cadherins is associated with tumourigenesis, in particular the expression of epithelial (E)-cadherin is reported to be reduced or lost in malignant cells. Colorectal cancer (CRC), a lethal and common type of cancer, is mainly comprised of adenocarcinomas. In many cases it is asymptomatic, and the survival of the patient depends on the time of diagnosis and the stage of tumour. The asymptomatic nature of colorectal cancer makes early diagnosis of this cancer difficult. In this context, the expression of E-cadherin as a biomarker to predict CRC progression is still in progress [2]. Besides reduced or absent E-cadherin expression, the redistribution pattern of E-cadherin from membrane to cytoplasm is also indicative of a more poorly differentiated form of CRC, which thereby facilitates tumour cell invasion and metastasis [1]. Multiple clinicopathological studies have associated E-cadherin with colorectal cancer. All these studies have linked reduced E-cadherin expression with loss of differentiation in colorectal cancer [3].

A lower 5-year survival rate in stage II CRC patients with reduced expression of E-cadherin was observed [4]. Furthermore, using small interfering RNA (siRNA)-mediated inhibition of E-cadherin expression, increased growth and metastatic invasion of colorectal cancer cells was noted [5]. The expression level of E-cadherin along with other molecules like $\beta$-catenin [6] and many more have been consistently used to predict the prognosis 
and outcome in CRC patients. Despite advances in use of E-cadherin expression in colorectal cancer, its use as a prognostic marker has been inconclusive, in particular in European populations [7]. In India both colon and rectal cancer are prevalent; among the most common cancers in males, colon cancer ranks $8^{\text {th }}$ followed by rectal cancer, whereas among females colon cancer ranks $9^{\text {th }}$, and lower incidences of rectal cancer are observed in females [8]. Very few Indian studies have explored the prognostic implication of immunohistochemical analysis of E-cadherin in Indian colorectal cancer population.

\section{Aim}

The main objective of this study was to evaluate the role of E-cadherin as a prognostic marker by analysing its intensity of expression in colorectal carcinomas.

\section{Material and methods \\ Patients and tumour samples}

In this retrospective study, paraffin blocks of 100 colon cancer specimens were obtained from the pathology department of the Tertiary Care hospital in South India between January 2013 and January 2016. The demographic details included age and gender, and pathological details included grade of tumour, stage of tumour, site of cancer, tumour invasion, and metastasis. Before commencing the present study, permission of the institutional ethics committee (Reference no: CSP-MED/14/ OCT/19/199) was obtained.

\section{Immunohistochemical (IHC) staining}

\section{Preparation of slides}

The obtained paraffin blocks were made on samples from tumour areas along with adjacent normal tissue from colectomy specimens received in the department. The sections were deparaffinised with xylene for $5 \mathrm{~min}$ and then treated twice with absolute alcohol for $1 \mathrm{~min}$ each and finally rinsed with water. The staining was performed using Harris Haematoxylin for $5 \mathrm{~min}$, and excess stain was washed off with water twice. The section was then stained in $1 \%$ aqueous Eosin $Y$ for 2 min and then washed in running tap water for $1 \mathrm{~min}$. The stained slide was dehydrated by making 3 changes of absolute alcohol. The slides were then rinsed with $x y-$ lene twice and were mounted with DPX. IHC for E-Cadherin was done on the sections along with internal control, which is the normal colonic epithelium.

\section{E-cadherin staining}

For the E-cadherin staining, the prepared unstained sections were placed on the 0.1 Poly-L-lysine coated slides. The sections were circled and labelled with a diamond pencil. The sections were then placed in a slide warmer at $60^{\circ} \mathrm{C}$ for $30 \mathrm{~min}$. The slides were then transferred to xylene ( 2 baths) for 5 min each and then placed in absolute alcohol twice. The slides were then rinsed in running water and placed in $0.01 \mathrm{M}$ citrate buffer $\mathrm{pH} 6.0$ for pressure cooker heating. The pressure cooker was set at an operating pressure of 103/ $115 \mathrm{kPa}$ to ensure $120^{\circ} \mathrm{C}$ temperature at full pressure. The sections were then retrieved using a target retrieval buffer at room temperature for 15-20 min in the pressure cooker. The solution was boiled and immediately cooled by placing the pressure cooker in cold running water. The slides were transferred to Tris buffer solution (Tris hydroxyl methyl amino methane), and the endogenous peroxidase activity was blocked by using $3 \%$ hydrogen peroxide. The slides were then rinsed in distilled water for $5 \mathrm{~min}$. Again, the slides were rinsed in Tris buffer solution, and power block was added for $10 \mathrm{~min}$. The slides were then incubated with primary antibody (E-cadherin, EP700Y, rAb; Cell Marque) for $1 \mathrm{~h}$. The slides were then rinsed in Tris buffer solution and incubated with super enhancer. Again, the slides were rinsed in Tris buffer solution. The slides were then incubated with Horseradish Peroxidase (HRP)-polymer and then again rinsed in Tris buffer solution. The excess buffer was then wiped off, and the slides were incubated with substrate/chromogen for $5 \mathrm{~min}$. The slides were then rinsed in distilled water and counter-stained with Haematoxylin for 3 min. Finally, the slides were dehydrated and mounted.

\section{Evaluation (scoring) of E-cadherin staining}

The intensity of E-cadherin was read semi-quantitatively based on the immunostaining intensity and the percentage of E-cadherin-positive cells. Based on the Allred scoring system, 4 categories of staining $(0,1$, 2 , and 3 ) ranging from none (negative) to strong were used. Zero $(0)$ referred to negative/none with zero positive cells, 1 - weak with < 1 positive cell, 2 - intermediate with 1-10 positive cells, and 3-strong with 11-33 positive cells.

\section{Ethics statement}

All procedures performed in this study were approved by the Institutional Ethics Committee (IEC), (CSP-MED/14/OCT/19/199) in accordance with the ICMR guidelines on Biomedical Research in Human beings. Formal written informed consent was waived by the IEC.

\section{Statistical analysis}

For all the statistical analysis Statistical Package for the Social Sciences (SPSS) 24.0v software was used. 
The clinical profile data were presented as a frequency distribution. $\chi^{2}$ tests were used to analyse the expression and association of E-cadherin with the clinical and pathological features of the CRC patients. A $p$-value less than 0.05 was considered statistically significant.

\section{Results}

In this study, a total of 100 patients diagnosed with CRC were included. The clinical profile including demographic and histopathological details of CRC are presented in Table I. The majority of patients belonged to the age group 41-60 years (45\%), and CRC was more predominant in males (58\%). The most common site

Table I. Patients and their tumour characteristics

\begin{tabular}{|c|c|}
\hline Characteristics & $\%$ \\
\hline \multicolumn{2}{|l|}{ Age [years]: } \\
\hline $21-40$ & 15.0 \\
\hline $41-60$ & 45.0 \\
\hline $61-80$ & 39.0 \\
\hline$>81$ & 1.0 \\
\hline \multicolumn{2}{|l|}{ Sex: } \\
\hline Male & 58.0 \\
\hline Female & 42.0 \\
\hline \multicolumn{2}{|l|}{ Size of tumour [cm]: } \\
\hline$<5$ & 37.0 \\
\hline $5-10$ & 55.0 \\
\hline$>10$ & 8.0 \\
\hline \multicolumn{2}{|l|}{ Sites: } \\
\hline Colon & 62.0 \\
\hline Rectum & 38.0 \\
\hline \multicolumn{2}{|l|}{ Histological type: } \\
\hline Adenocarcinoma & 67.0 \\
\hline Mucinous adenocarcinoma & 27.0 \\
\hline Signet ring cell carcinoma & 2.0 \\
\hline Mucinous adenocarcinoma with signet & 3.0 \\
\hline Adenocarcinoma with signet & 1.0 \\
\hline \multicolumn{2}{|l|}{ Tumour differentiation pattern: } \\
\hline Well differentiated & 13.0 \\
\hline Moderately differentiated & 74.0 \\
\hline Poorly differentiated & 13.0 \\
\hline \multicolumn{2}{|l|}{ Primary tumour (T status): } \\
\hline PT1 & 1.0 \\
\hline PT2 & 42.0 \\
\hline PT3 & 42.0 \\
\hline PT4 & 15.0 \\
\hline
\end{tabular}

of the tumour was the colon (62\%) as compared to the rectum (38\%), and the histological type was adenocarcinoma including mucinous type with a size range of $5-10 \mathrm{~cm}$ (55\%). The majority of the tumours were moderately differentiated (74\%), followed by poorly differentiated in $13 \%$. The extent of invasion of the tumour up to the muscularis propria (pT2) was noted in $42 \%$ of samples, and into the peri colorectal tissues in $42 \%$ (pT3). Lymphovascular invasion (LVI) was observed in $30 \%$ of patient samples. Furthermore, in $27 \%$ of samples CRC was found in more than one lymph node (N1), whereas in $13 \%$ of samples more than 4 lymph nodes were involved (N2). With respect to metastasis, many

\section{Characteristics}

$\%$

Lymph node involvement ( $\mathrm{N}$ status):

\begin{tabular}{ll}
\hline NX & 6 \\
\hline N0 & 54 \\
\hline N1 & 27 \\
\hline N2 & 13
\end{tabular}

\section{Metastasis:}

\begin{tabular}{lc}
\hline M0 & 95.0 \\
\hline M1 & 2.0 \\
\hline MX & 3.0
\end{tabular}

Presence of lymph vascular invasion:

Yes 30.0

No $\quad 69.0$

Not mentioned

1.0

Presence of perineural invasion:

\begin{tabular}{ll}
\hline Yes & 15.0 \\
\hline
\end{tabular}

No $\quad 84.0$

Not mentioned

1.0

Involvement of radial margin:

\begin{tabular}{lc}
\hline Yes & 6.0 \\
\hline No & 93.0 \\
\hline Not mentioned & 1.0 \\
\hline
\end{tabular}

Tumour deposit:

\begin{tabular}{lc}
\hline Yes & 5.0 \\
\hline No & 94.0 \\
\hline Omental present & 1.0
\end{tabular}


Table II. Correlation between E-cadherin expression and clinic-pathological parameters

\begin{tabular}{|c|c|c|c|c|c|c|c|}
\hline \multirow[t]{2}{*}{ Parameter } & \multirow{2}{*}{$\begin{array}{c}\text { Total } \\
(N=100)\end{array}$} & \multicolumn{4}{|c|}{ E cadherin expression - carcinoma intensity } & \multirow[t]{2}{*}{$\chi^{2}$} & \multirow[t]{2}{*}{$P$-value } \\
\hline & & 0 & $1+$ & $2+$ & $3+$ & & \\
\hline \multicolumn{8}{|l|}{ Age [years]: } \\
\hline$<45$ & 26 & $4(15.4)$ & $10(38.5)$ & $8(30.8)$ & $4(15.4)$ & \multirow[t]{2}{*}{4.620} & \multirow[t]{2}{*}{0.202} \\
\hline$>45$ & 74 & $6(8.1)$ & $17(23.0)$ & $29(39.2)$ & $22(29.7)$ & & \\
\hline \multicolumn{8}{|l|}{ Sex: } \\
\hline Male & 58 & $9(15.5)$ & $15(25.9)$ & $20(34.5)$ & $14(24.1)$ & \multirow[t]{2}{*}{4.690} & \multirow[t]{2}{*}{0.196} \\
\hline Female & 42 & $1(2.4)$ & $12(28.6)$ & $17(40.5)$ & $12(28.6)$ & & \\
\hline \multicolumn{8}{|l|}{ Size of tumor $[\mathrm{cm}]$ : } \\
\hline$<5$ & 37 & $5(13.5)$ & $12(32.4)$ & $11(29.7)$ & $9(24.3)$ & \multirow[t]{3}{*}{3.393} & \multirow[t]{3}{*}{0.758} \\
\hline $5-9.99$ & 55 & $4(7.3)$ & $13(23.6)$ & $22(40.0)$ & $16(29.1)$ & & \\
\hline$>10$ & 8 & $1(12.5)$ & $2(25.0)$ & $4(50.0)$ & $1(12.5)$ & & \\
\hline \multicolumn{8}{|l|}{ Sites: } \\
\hline Rectum & 38 & $2(5.3)$ & $10(26.3)$ & $19(50.0)$ & $7(18.4)$ & \multirow[t]{2}{*}{5.539} & \multirow[t]{2}{*}{0.136} \\
\hline Colon & 62 & $8(12.9)$ & $17(27.4)$ & $18(29.3)$ & $19(30.6)$ & & \\
\hline \multicolumn{8}{|c|}{ Tumour invasion (T status): } \\
\hline PT1 & 1 & $0(0.0)$ & $0(0.0)$ & $0(0.0)$ & $1(100.0)$ & \multirow[t]{4}{*}{6.793} & \multirow[t]{4}{*}{0.659} \\
\hline PT2 & 42 & $2(4.8)$ & $10(23.8)$ & $19(45.2)$ & $11(26.2)$ & & \\
\hline PT3 & 42 & $6(14.3)$ & $12(28.6)$ & $14(33.3)$ & $10(23.8)$ & & \\
\hline PT4 & 15 & $2(13.3)$ & $5(33.3)$ & $4(26.7)$ & $4(26.7)$ & & \\
\hline \multicolumn{8}{|c|}{ Lymph node involvement ( $\mathrm{N}$ status): } \\
\hline NX & 6 & $1(16.7)$ & $0(0.0)$ & $4(66.7)$ & $1(16.7)$ & \multirow[t]{4}{*}{22.82} & \multirow[t]{4}{*}{0.007} \\
\hline NO & 54 & 7 (13.0) & $12(22.2)$ & $20(37.0)$ & $15(27.8)$ & & \\
\hline N1 & 27 & $0(0.0)$ & $6(22.2)$ & $13(48.1)$ & $8(29.6)$ & & \\
\hline $\mathrm{N} 2$ & 13 & $2(15.4)$ & $9(69.2)$ & $0(0.0)$ & $2(15.4)$ & & \\
\hline \multicolumn{8}{|l|}{ Metastasis: } \\
\hline MO & 95 & $26(27.4)$ & $36(37.9)$ & $24(25.3)$ & $9(9.5)$ & \multirow[t]{3}{*}{4.196} & \multirow[t]{3}{*}{0.650} \\
\hline $\mathrm{M} 1$ & 2 & $0(0.0)$ & $1(50.0)$ & $1(50.0)$ & $0(0.0)$ & & \\
\hline$M X$ & 3 & $1(33.3)$ & $0(0.0)$ & $1(33.3)$ & $1(33.3)$ & & \\
\hline Tumour differentiatior & attern: & & & & & & \\
\hline $\begin{array}{l}\text { Poorly } \\
\text { differentiated }\end{array}$ & 13 & $5(38.5)$ & $4(30.8)$ & $2(15.4)$ & $2(15.4)$ & 17.131 & 0.009 \\
\hline $\begin{array}{l}\text { Moderately } \\
\text { differentiated }\end{array}$ & 74 & $3(4.1)$ & $19(25.7)$ & $30(40.5)$ & $22(29.7)$ & & \\
\hline Well differentiated & 13 & $2(15.4)$ & $4(30.8)$ & $5(38.5)$ & $2(15.4)$ & & \\
\hline Cytoplasm or membra & & & & & & & \\
\hline Negative/NA & 10 & 10 & $0(0.0)$ & $0(0.0)$ & $0(0.0)$ & 104.32 & $<0.001$ \\
\hline Membrane & 10 & $0(0.0)$ & $4(40.0)$ & $3(30.0)$ & $3(30.0)$ & & \\
\hline Cytoplasm & 37 & $0(0.0)$ & $14(37.8)$ & $15(40.5)$ & $8(21.6)$ & & \\
\hline $\begin{array}{l}\text { Membrane and } \\
\text { cytoplasm }\end{array}$ & 43 & $0(0.0)$ & $9(20.9)$ & $19(44.2)$ & $15(34.9)$ & & \\
\hline
\end{tabular}


samples showed that the cancer had not spread to distant parts of the body (95\%). Other clinical features like perineural invasion (15\%), involvement of radial margin (6\%), and tumour deposit (5\%) had lower frequency.

\section{Association of E-cadherin expression with clinicopathological parameters}

Table II exemplifies the association of E-cadherin staining with clinical as well as pathological parameters of colorectal cancer patients. An insignificant association $(p>0.05)$ was observed between E-cadherin expression and parameters such as age $\left(\chi^{2}=4.620\right)$, gender $\left(\chi^{2}=4.690\right)$, and size of tumour $\left(\chi^{2}=3.393\right)$. Additionally, E-cadherin expression showed statistically insignificant correlation with sites of cancer $\left(\chi^{2}=5.539, p>0.05\right)$ and tumour invasion (T-status) $\left(\chi^{2}=6.793\right)$. The correlation of E-cadherin expression with lymph node involvement was statistically significant, as revealed by a $\chi^{2}$ value of 22.82 ( $p<0.01$ ). About $54 \%$ samples showed no lymph node invasion.
Lymph node involvement of N1 type with invasion of $1-3$ lymph nodes was found in $27 \%$ of samples, and about $13 \%$ samples had lymph node invasion in more than 4 nodes (N2). The association between E-cadherin expression and distant organ metastasis was insignificant $\left(\chi^{2}=4.196, p>0.05\right)$. In the majority of samples (95\%) there was no distant organ metastasis, and 2 samples showing metastasis revealed weak to intermediate E-cadherin staining. However, E-cadherin expression was significantly correlated with tumour differentiation pattern $\left(\chi^{2}=17.131, p<0.01\right)$. Larger numbers of samples were moderately differentiated ( $n=74 \%$ ). Out of 13 poorly differentiated carcinomas, 5 had no E-cadherin staining and 4 had a weak staining. In normal cells, membranous staining of E-cadherin was found in $83 \%$ of samples. Figure 1 depicts the representative samples of E-cadherin immunostaining. In carcinoma cells, the association between E-cadherin expression intensity and IHC expression pattern was found to be significant $\left(\chi^{2}=104.32, p<0.01\right)$. Mixed $\mathrm{E}$-cadherin expression
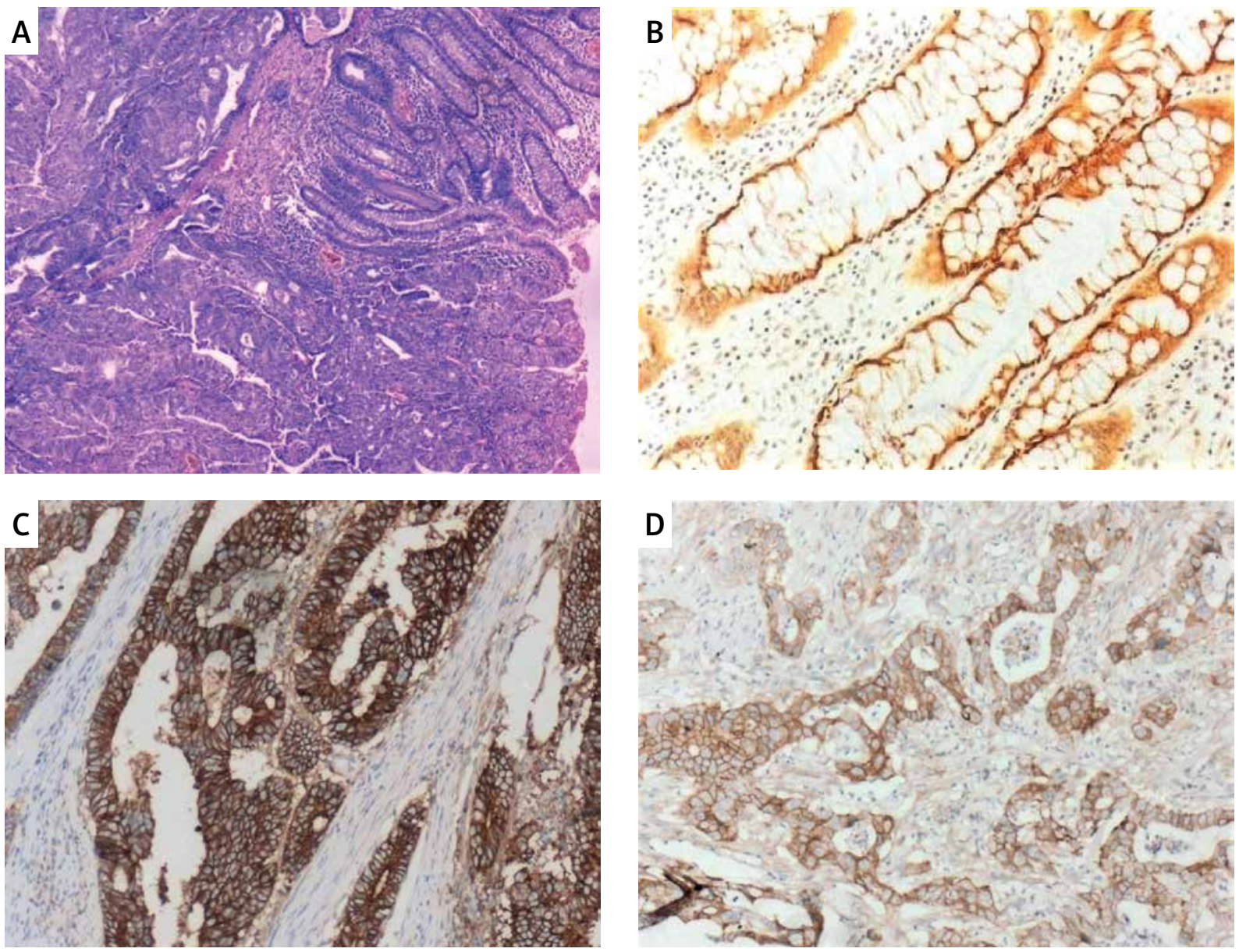

Figure 1. A - Adenocarcinoma with adjacent normal colonic tissue (H\&E; 40x). B - Normal colonic epithelium with membrane - E-cadherin immunostaining (200x). C - Adenocarcinoma with E-cadherin immunostaining - score 3+ (IHC, 200x). D - Adenocarcinoma with E-cadherin staining - score 2+ (IHC, 200x) 


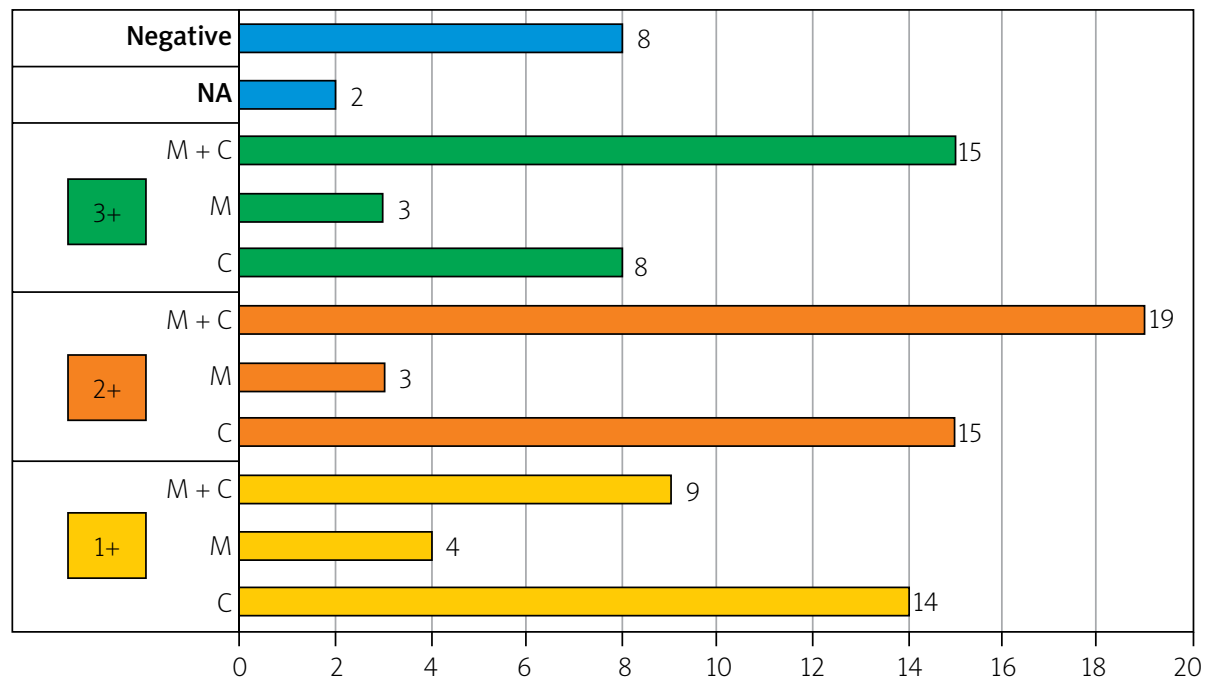

Figure 2. IHC pattern with intensity correlation $(n=100)$

$M$ - membrane, $C$-cytoplasm, $M+C$ - membrane and cytoplasm.

(membrane and cytoplasmic) ( $n=43 \%)$ was the predominant one followed by cytoplasmic E-cadherin expression (37\%), suggestive of the presence of a tumour (Figure 2 ).

\section{Discussion}

Among many biomarkers, E-cadherin expression is associated with tumour progression [2]. In general, the loss or heterogeneous expression of E-cadherin is related to advanced stages of colorectal cancer, progression and differentiation, metastasis, and poor outcomes like low survival, thereby making it an important clinicopathological marker [9].

In the present study, the E-cadherin expression did not correlate with the age and gender of patients. Similarly, with respect to tumour characteristics, negative E-cadherin expression was not correlated to size, site, or grade of tumour. In the literature, the correlation of E-cadherin expression with age, gender, and tumour size is inconsistent. The present findings agree with observations reported by Khoursheed et al. [10], but they contrast with another study wherein the author found significant association between E-cadherin membrane expression and the age factor of the colon carcinoma patients [6]. The link between E-cadherin expression and tumour invasion was not observed in this study, in contrast to the observations of Hong et al. [11], who showed an inverse correlation between E-cadherin and depth of tumour invasion, indicating decreased E-cadherin expression with increased depth of tumour invasion, and this was related to higher Slug expression, which was responsible for the inhibition of E-cadherin expression [11].

Higher or positive expression of E-cadherin in epithelial cells of well-differentiated tumours as well as moderately differentiated CRC has been observed by many researchers $[10,12]$. Low E-cadherin expression is linked to poor survival outcomes. A similar line of inference can be drawn from our results also, wherein negative to weak E-cadherin expression was found in poorly differentiated colon carcinoma cells, indicating that loss of expression of E-cadherin protein can result in poorly differentiated tumours, as observed in other cancers such as non-small cell carcinoma of the lung and carcinoma of the rectum $[13,14]$. Using a multiple-punch tissue microarray, it was shown that among 21 tumour-related factors, E-cadherin was independently regarded as a predictor of lymph node $(N)$ stage in $\mathrm{CRC}$, indicative of its expression in risk stratification of patients with colorectal cancer [15]. A mixed (membrane and cytoplasm) E-cadherin expression pattern in CRC with metastatic lymph nodes agreed with the present observation [16].

In normal colorectal epithelial cells, E-cadherin expression is predominantly distributed in membranes of borders of cells. A poor survival rate is predicted in CRC patients with increased cytoplasmic E-cadherin expression, which suggests cytoplasmic expression of E-cadherin as a prognostic factor for colorectal cancer [17]. Likewise, according to Elzagheid et al., cytoplasmic expression of E-cadherin in primary colorectal tumour reduces the chances of survival, and mixed staining (membrane and cytoplasmic) of E-cadherin was observed in patients who later developed metastases in the liver [18]. This could be indicative of slow progressive metastasis of colorectal cancer. In concordance with our findings, a similar observation on loss of normal membranous expression and the presence of cyto- 
plasmic and mixed staining in tumour tissues was made by Khoursheed et al. [10]. Loss of E-cadherin expression can induce an epithelial-to-mesenchymal transition and result in tumour invasiveness. In general, lower expression of E-cadherin is found in metastasized CRC, which could be due to Snail2-mediated epigenetic suppression of E-cadherin levels during metastasis [19]. According to a meta-analytic review of 27 studies, in the Asian population low or absent E-cadherin expression detection by IHC was observed in metastatic tumours [7]. In the present study, because there were few samples with distant metastasis, little could be extrapolated on E-cadherin expression during metastasis; however, El Gehani et al. observed no link between E-cadherin expression and distant metastasis, which is in agreement with the findings of this study [20]. Early screening of changes in the colon is key in reducing malignancy and mortality in colorectal cancer patients. In this regard, minimally invasive colonoscopy is the gold standard technique; however, non-invasive and low-cost faecal screening tests like the immunochemical Faecal Occult Blood Test (iFOBT) and the guaiac faecal occult blood test (gFOBT) are recommended every year for the earliest screening of colorectal cancer [21].

The present study provides an insight into the use of IHC of E-cadherin expression in tumour cells as a prognostic marker. However, the data from this study have limitations. The small sample size of 100 patients, and all being from a single centre could limit the generalization of findings. Furthermore, the expression of E-cadherin binding partner $\beta$-catenin was not tested. Also, the probable disruption in the signalling pathway due to E-cadherin loss was not explored. Therefore, a large and prospective study will further strengthen the findings of this study.

\section{Conclusions}

This study was performed to analyse the utility of IHC expression of E-cadherin as a prognostic marker in the detection of colorectal cancer. A reduction in E-cadherin expression was found in samples with colorectal cancer, and it was predominant in poorly differentiated cells. A shift from membranous staining of E-cadherin in normal cells to cytoplasmic or mixed membrane and cytoplasmic staining in cancer cells was observed. The low/weak E-cadherin expression indicated invasiveness of $C R C$ to regional lymph nodes and the progressive nature of colorectal cancer, thereby supporting the use of E-cadherin as a prognostic biomarker for colorectal carcinomas.

\section{Conflict of interest}

The authors declare no conflict of interest.

\section{References}

1. Buda A, Pignatelli M. E-cadherin and the cytoskeletal network in colorectal cancer development and metastasis. Cell Commun Adhesion 2011; 18: 133-43.

2. Christou N, Perraud A, Blondy S, et al. E-cadherin: a potential biomarker of colorectal cancer prognosis. Oncol Letters 2017; 13: 4571-6.

3. Dorudi S, Sheffield JP, Poulsom R, et al. E-cadherin expression in colorectal cancer an immunocytochemical and in situ hybridization study. Am J Pathol 1991; 142: 981-6.

4. Bruun J, Kolberg M, Nesland JM, et al. Prognostic significance of $\beta$-catenin, E-cadherin, and SOX9 in colorectal cancer: results from a large population-representative series. Front Oncol 2014; 4: 118.

5. Chen X, Wang Y, Xia H, et al. Loss of E-cadherin promotes the growth, invasion and drug resistance of colorectal cancer cells and is associated with liver metastasis. Mol Biol Rep 2012; 39: 6707-14.

6. Ahmad A, Hahn-Stromberg V. Expression of $\beta$-catenin and E-cadherin, their clinical significance and association with complexity index of colon carcinoma. Adv Genet Engin 2016; 5: 3.

7. He X, Chen Z, Jia M, et al. Downregulated E-cadherin expression indicates worse prognosis in Asian patients with colorectal cancer: evidence from meta-analysis. PLoS One 2013; 8: e70858.

8. Sirohi B, Shrikhande SV, Perakath B, et al. Indian Council of Medical Research consensus document for the management of colorectal cancer. Indian J Med Paediatr Oncol 2014; 35 : 192-6.

9. Mohri Y. Prognostic significance of E-cadherin expression in human colorectal cancer tissue. Surgery Today 1997; 27: 606-12.

10. Khoursheed MA, Mathew TC, Makar RR, et al. Expression of E-cadherin in human colorectal cancer. Surgeon 2003; 1: 86-91.

11. Hong R, Choi DY, Lim SC, et al. The differential expressions of the epithelial-mesenchymal transition regulator, slug and the cell adhesion molecule, E-cadherin in colorectal adenocarcinoma. Korean J Pathol 2008; 42: 351-7.

12. Yun JA, Kim SH, Hong HK, et al. Loss of E-cadherin expression is associated with a poor prognosis in stage III colorectal cancer. Oncology 2014; 86: 318-28.

13. Liu D, Huang C, Kameyama K, et al. E-cadherin expression associated with differentiation and prognosis in patients with non-small cell lung cancer. Ann Thorac Surg 2001; 71: 949-54.

14. Kanazawa N, Oda T, Gunji N, et al. E-cadherin expression in the primary tumors and metastatic lymph nodes of poorly differentiated types of rectal cancer. Surgery Today 2002; 32: 123-8.

15. Karamitopoulou E, Zlobec I, Patsouris ES, et al. Loss of E-cadherin independently predicts the lymph node status in colorectal cancer. Pathology 2011; 43: 133-7.

16. Palaghia MĂ, Mihai C̆́, Lozneanu L, et al. E-cadherin expression in primary colorectal cancer and metastatic lymph nodes. Rom J Morphol Embryol 2016; 57: 205-9.

17. Bendardaf R, Sharif-Askari FS, Sharif-Askari NS, et al. Cytoplasmic E-cadherin expression is associated with higher tumour level of VEGFA, lower response rate to irinotecan-based 
treatment and poorer prognosis in patients with metastatic colorectal cancer. Anticancer Res 2019; 39: 1953-7.

18. Elzagheid A, Algars A, Bendardaf R, et al. E-cadherin expression pattern in primary colorectal carcinomas and their metastases reflects disease outcome. World J Gastroenterol 2006; 12 : 4304-9.

19. Hu Y, Dai M, Zheng Y, et al. Epigenetic suppression of E-cadherin expression by Snail2 during the metastasis of colorectal cancer. Clin Epigenetics 2018; 10: 154.

20. El Gehani K, Aljafil R, El Hasadi I, et al. Loss of E-cadherin expression in colorectal carcinoma and its prognostic significance. Acta Sci Gastrointest Disord 2019; 2: 03-08.

21 . Kowalik J. Faecal tests in the early detection of colorectal cancer. Gastroenterology Rev 2020; 15: 200-6.

Received: 26.04.2021

Accepted: 28.05 .2021 